\title{
RELACIÓN ENTRE AUTOEFICACIA, AUTOESTIMA, ASERTIVIDAD, Y RENDIMIENTO ACADÉMICO, EN ESTUDIANTES QUE INGRESARON A TERAPIA OCUPACIONAL, EL AÑO 2010
}

\section{RELATIONSHIP BETWEEN SELF-EFFICACY, SELF-ESTEEM, ASSERTIVENESS, AND ACADEMIC PERFORMANCE, IN STUDENTS WHO STARTED IN 2010, THE CAREER OF OCCUPATIONAL THERAPY}

\author{
Aluicio G., Anaís ${ }^{1}$ y Revellino, Mónica ${ }^{2}$
}

\section{RESUMEN}

Se presentan los resultados de investigación de un estudio correlacional, realizado con una muestra de los estudiantes de ingreso en 2010, a la carrera Terapia Ocupacional, de la Universidad San Sebastián, en su sede de Santiago. Se establecen las relaciones entre autoestima, asertividad y autoeficacia, así como la vinculación de éstas con rendimiento académico. Se concluye que existe una correlación significativa entre las variables autoestima, asertividad y autoeficacia; pero no así entre éstas y rendimiento académico, pudiéndose verificar solamente una vinculación entre ellas, pero sin determinar el grado de la misma. Se hipotetiza al respecto de las posibles explicaciones de estos resultados y se contribuye con sugerencias metodológicas, para aportar al desarrollo profesional de los futuros terapeutas ocupacionales.

Palabras claves: autoestima, asertividad, autoeficacia, rendimiento académico, estudiantes universitarios.

\begin{abstract}
This study described the existent relationship between self-efficacy, self-esteem and assertiveness, and between these three variables and academic performance, in 43 students of both sexes, who started in 2010, the career of Occupational Therapy at the University San Sebastian, in Santiago de Chile. As a quantitative research, with a non-experimental and correlational design, it had the longing to establish relations between variables in study, but without determining causality between them. To this end, the procedures used were the evaluation with three self-administrated tests, as data collection instruments, that specifically measured levels of self-esteem, assertiveness and self-efficacy (Coopersmith self-esteem inventory, Rathus assertiveness scale and Scale of generalized self-efficacy); with its subsequent tabulation. Besides, was considered the score average obtained by these students in the first year of their career. The analysis of these results showed that there is a significant correlation between the variables self-esteem, assertiveness and self-efficacy; but not as well between these and academic performance. Although, was verified a relation between these variables but not the extent of it. It is hypothesized about the possible explanations for these results, and suggestions are provided.
\end{abstract}

Key words: self-esteem, assertiveness, self-efficacy, academic performance, college students.

\footnotetext{
${ }^{1}$ Psicóloga, Magíster en Educación y Formación Universitaria. Docente de la carrera Terapia Ocupacional, en la Universidad San Sebastián. Bellavista no7. (2) 915 4530. aaluicio@docenteuss.cl 2 Psicopedagoga. Magíster en Educación y Formación Universitaria.
} 


\section{INTRODUCCIÓN}

En un mundo en el que crecientemente se atribuye una vital importancia a las competencias blandas, pero en el que sigue siendo priorizado el conocimiento medible, resulta relevante establecer las posibles relaciones entre la mente emocional y la mente intelectual. Asimismo, las competencias emocionales y relacionadas con la personalidad son especialmente apreciables en las profesiones en las que la atención al usuario, cliente o paciente es una de las principales tareas del profesional, como es el caso del Terapeuta Ocupacional. En la atención a sus pacientes, el profesional debe mostrar la capacidad de controlar sus propias emociones y detectar, interpretar y manejar correctamente las emociones de los demás, ya que éstas son un indicador de las necesidades del paciente (1).

Teniendo en cuenta lo anterior, cada vez resulta más evidente la necesidad de que los profesionales en formación desarrollen a lo largo de su carrera, no sólo competencias duras o cognitivas, sino además, habilidades sociales, ya que éstas influirán en gran medida en su desarrollo profesional y empleabilidad. En este sentido, comenta Martínez (2) que hay personas que son cognoscitivamente muy inteligentes, pero que al carecer de inteligencia emocional, terminan trabajando para otros con cocientes intelectuales inferiores, pero con una inteligencia emocional superior a la suya. Sin afán de profundizar, se considera oportuno brindar una pincelada, que permita la comunicación efectiva en lo que respecta al concepto de inteligencia emocional. El término se refiere a la capacidad de reconocer los propios sentimientos, los sentimientos de los demás, motivarnos y manejar, adecuadamente, las relaciones que sostenemos con los otros y con nosotros mismos (2). En resumen, son un grupo de competencias referidas a las relaciones sociales, que se expresan en varios ámbitos de la vida, especialmente en aquellos en que los individuos interactúan con otras personas, como es el caso del ambiente laboral y académico.

Por otra parte, la inteligencia "intelectual", o más comúnmente relacionada con las

habilidades cognitivas, se ha valorado tradicionalmente a través del rendimiento académico. Sin embargo, éste como predictor del éxito laboral, no está exento de críticas, por su confiabilidad especialmente en contextos académicos alejados de la realidad laboral; y a pesar de que en muchos casos, suele dar una visión general del aprovechamiento del estudiante de las instancias de aprendizaje, se aprecia que necesitan ser tomadas en consideración otras variables, en este caso comunicacionales, que aportarían elementos relevantes al respecto de la calidad del aprendizaje. Esto, considerando que en la Educación Superior se prepara a los futuros profesionales para desempeñarse en áreas laborales (como el sector de la salud) que requiere no sólo conocimiento tácito, sino además, explícito.

Existen varias habilidades que se han relacionado con el éxito laboral, entre ellas está la asertividad. Según Roberto Opazo, "una persona es asertiva cuando tiene un estilo de comunicación abierto y desenvuelto, cuando es capaz de expresar en forma directa lo que piensa y siente, cuando es capaz de defender con decisión y firmeza sus opiniones y derechos, sin atropellar los derechos de los demás" (3). La influencia de esta habilidad 
en los resultados de las evaluaciones académicas parece estar relacionada con la capacidad de los estudiantes para exponer sus ideas adecuadamente, para argumentar sus puntos de vista, o para ejercer su capacidad crítica, lo cual pudiera impulsar una conducta adecuada en situaciones de trabajo cognitivo. El comportamiento poco asertivo pudiera ser un obstáculo para el buen desempeño, por ejemplo, en evaluaciones orales o en aquellas que requirieran del trabajo en equipo. Sin embargo, las investigaciones de varios autores demuestran la importancia de la asertividad para el desempeño laboral, especialmente en las carreras del área de la salud (4) (5). También, un estudio en La Habana, muestra que en el área de la salud, el 30\% de los profesionales presenta indicadores de Síndrome de Burnout, y el 50\% de estrés; ambos padecimientos se relacionan con el área de desempeño profesional, lo que estaría confirmando que el área de la salud es una de las más demandantes en lo que respecta a recursos psicológicos (6). Se entiende, que un profesional psicológicamente inestable no está capacitado para brindar una adecuada atención a sus pacientes. Otros autores muestran cómo, en las personas con un alto nivel de asertividad, la ocurrencia de enfermedades somáticas asociadas al estrés es menor, propiciando una mejor calidad de vida y así un mejor desempeño laboral (7). Estas evidencias son preocupantes en un contexto en el que se premia, fundamentalmente, el rendimiento académico. Si los profesionales de la salud que se gradúan en la actualidad, manejan muy bien los contenidos de las materias propias de las carreras, pero no desarrollan las habilidades sociales necesarias para el tratamiento adecuado con sus pacientes, probablemente no serán profesionales de calidad. Así, surge la preocupación al respecto de que el rendimiento académico entonces no sea un buen predictor del éxito profesional. Por esta razón, se considera relevante incluir la medida de asertividad en este estudio, pues, en caso de existir una correlación positiva entre ambas variables, se pondría en evidencia la integralidad de las evaluaciones que se llevan a cabo durante la carrera Terapia Ocupacional, y en caso contrario, estaría mostrando un área a mejorar en el desarrollo integral de los estudiantes de la carrera.

Otro concepto muy relacionado con la capacidad de manejo social es la autoestima, Neva Milicic la conceptualiza como "la suma de juicios que una persona tiene de sí misma; es decir, lo que la persona se dice a sí misma sobre sí misma" (8). Teniendo en cuenta esta definición, se puede decir que las personas, en el proceso de construcción de su identidad, incorporan creencias acerca de sí mismos, las cuales conforman esquemas cognitivos, que rigen pautas de conducta, y suelen tener como objetivo ser coherentes con dichas creencias (9). Es así, que si un estudiante posee una pobre visión sobre sí mismo, probablemente sus puntuaciones no sean altas; así lo evidencian diversas investigaciones (10) (11).

Relacionado estrechamente con el anterior, el concepto de autoeficacia describe el fenómeno de creer en las capacidades personales. "La autoeficacia sustenta la fe en la capacidad de realizar una determinada acción o producir un resultado deseado", dice Feldman (12). Albert Bandura, quien desarrolló en profundidad estudios relacionados con este tema, afirma que aquellas personas con un grado elevado de autoeficacia se plantean metas más elevadas, son más persistentes en el trabajo para conseguirlas y suelen lograr un mayor éxito que quienes tienen un grado de autoeficacia bajo (12). Dicho esto, resulta valioso entonces analizar la relación existente entre la autoeficacia y el rendimiento académico. 
En el caso de la autoestima y la autoeficacia, se hallan varios estudios que muestran que existe relación entre ellas (11) y de éstas con el rendimiento (13) (10) (14). Sin embargo, pocos autores revisan la relación entre estos indicadores del desarrollo de la personalidad y la asertividad, a la vez que analizan su relación con el rendimiento académico. Si bien Reyes (15) es una de ellos, dicha investigación fue realizada en un país distinto de Chile, por lo que resulta conveniente aportar datos empíricos al respecto de este tema, en el contexto educacional nacional; además, la investigación mencionada anteriormente no correlaciona asertividad con autoestima o autoeficacia, sino sólo con la variable rendimiento académico (15).

El contexto actual aún privilegia el desarrollo del contenido y no de la forma del aprendizaje. Este problema se muestra más claramente en las salas de clases, cuando se prioriza el manejo conceptual sobre el desarrollo de la personalidad de los estudiantes. Aquí se pone de manifiesto la visión dicotómica del ser humano, que aparentemente es visto como un "ser que piensa", obviando que además, es un "ser que siente". El aprendizaje debe ser visto como un proceso de crecimiento en todas las dimensiones de la experiencia de los estudiantes. En estos días, se necesita evidencia, en el contexto universitario, para motivar un cambio de paradigma, que se traduzca en la creación e implementación de programas transversales de desarrollo integral en los profesionales, especialmente aquellos que se desempeñarán en el área de la salud. Al establecer las posibles relaciones entre variables vinculadas con la personalidad y el rendimiento académico se pretende poner de manifiesto la importancia de implementar programas educativos integrales y aportar datos empíricos que avalen la necesidad de analizar multifactorialmente el desarrollo de los estudiantes en las salas de clases.

De esta manera, se manejó como hipótesis que existiría una relación directa entre las variables personológicas en estudio, y de éstas con el rendimiento académico. Este planteamiento de base se relaciona con el propósito de describir la relación existente entre la autoestima, la autoeficacia, la asertividad y el rendimiento académico, en los estudiantes que ingresaron en el 2010, a la carrera de Terapia Ocupacional, de la Universidad San Sebastián, de la sede Santiago de Chile.

\section{METODOLOGÍA}

Destacamos primeramente, para favorecer la comprensión la metodología utilizada y el análisis de los resultados alcanzados, que las hipótesis de investigación en las que se centró este estudio fueron:

$\mathrm{H}_{1}$ : A mayor autoestima, habrá mayor asertividad.

$\mathrm{H}_{2}$ : A mayor autoeficacia, habrá mayor asertividad.

$\mathrm{H}_{3}$ : A mayor autoestima, habrá mayor rendimiento académico.

$\mathrm{H}_{4}$ : A mayor autoeficacia, habrá mayor rendimiento académico.

$\mathrm{H}_{5}$ : A mayor asertividad, habrá mayor rendimiento académico.

La investigación se enmarcó en un enfoque cuantitativo positivista. Para fundamentar dicha afirmación se comienza por presentar algunas posiciones conceptuales y su correlación con nuestra experiencia. Tal necesidad surge, como lo indica Smith, (17) en 
aclarar las discrepancias existentes entre los paradigmas cuantitativo y cualitativo de la investigación socioeducativa, que se inician en el enfrentamiento de los supuestos filósofos del positivismo y del idealismo científico (17). Según Hurtado y Toro (19):

La investigación cuantitativa debe tener una concepción lineal, es decir, que haya claridad entre los elementos que conforman el problema, que tenga definición, limitarlos y saber con exactitud donde se inicia el problema, (...) también le es importante saber qué tipo de incidencia existe entre sus elementos.

Haciendo un acercamiento de la conceptualización del paradigma cuantitativo a las especificaciones de la investigación social-educativa, se coincide con Taylor y Bogdan, (17) en que la búsqueda principal de una investigación cuantitativa consiste, entre otros, en confrontar teorías y praxis, detectar discrepancias, analizar estadísticamente, establecer conexiones, generalizaciones y abstracciones.

Esto deja en evidencia las características propias del paradigma cuantitativo: su objetividad, a través de la medición controlada; el carácter empírico del elemento en estudio; el sostén que brinda la teoría al marco conceptual en el que se sostiene; y las características deductivas de las estrategias usadas en el estudio.

Es justamente desde dicha orientación que se pretendió comprobar las hipótesis de trabajo, llegando así a una descripción de las relaciones entre las variables en estudio (autoestima, asertividad, autoeficacia y rendimiento académico). Esto, a través de medición exhaustiva, objetiva y estática que permite el uso de la estadística como instrumento para el análisis de las relaciones entre las variables en estudio.

Esto último confirma además la perspectiva paradigmática positivista en la que esta investigación se basa, desde el momento en que se comprende la independencia existente entre los fenómenos sociales y los estados subjetivos de los sujetos participantes en él.

Este estudio fue de tipo correlacional, con un diseño de investigación transeccional, pues se limitó a establecer relaciones entre las variables en estudio, sin el afán de precisar sentido de causalidad, y las mismas son medidas en un único momento en el tiempo (19). El propósito fue evaluar la relación entre las cuatro variables en estudio: autoestima, asertividad, autoeficacia y rendimiento académico. El foco de esta investigación está en la relación entre dichas variables, medidas en un momento determinado y no en el estudio de ellas individualmente.

La población estuvo conformada por estudiantes universitarios, que ingresaron a la carrera Terapia Ocupacional en el año 2010, en la Universidad San Sebastián, en su sede Santiago. En este caso, la muestra estuvo conformada por los alumnos que continuaban sus estudios en dicha institución, y que asistieron a la sesión de toma de las pruebas; siendo éstos 43 sujetos de ambos sexos, quienes consintieron informadamente participar, constituyendo el $75,4 \%$ de la población en estudio.

\section{Variables en estudio:}

\section{a) Autoestima. Definición operacional e instrumento de medición.}

Esta variable es de tipo cuantitativa y se obtuvieron resultados de la misma a través del Inventario de Autoestima de Coopersmith, validado en Chile por Brinkmann y Garcés, 
en 1999 (20). La tabulación del instrumento permitió obtener un valor para cada una de las cinco escalas del test (General, Hogar, Social, Laboral y M -validez-), y una clasificación en baja, media o alta, según las normas publicadas por los autores antes mencionados. Lara, Cantú, Verduzco, Acevedo y Cortés, (21) reportan para el cociente de confiabilidad encontrado al usar el alfa de Cronbach un valor de 0,81.

\section{b) Asertividad. Definición operacional e instrumento de medición.}

El nivel de asertividad fue medido, cuantitativamente, a través de la Escala de Asertividad de Rathus y los resultados se obtienen de acuerdo a los seis tipos de respuestas (Totalmente verdadero; Verdadero; Más verdadero que falso; Más falso que verdadero; Falso y Totalmente falso) que el sujeto puede seleccionar para contestar a treinta afirmaciones, teniendo presentes categorías descriptivas de sí mismo. Así, se obtuvieron rangos de respuestas que están clasificados en los niveles de asertividad (baja, media o alta), en el que se encuentra el individuo (22).

La escala de Rathus presenta buenas correlaciones con otros inventarios de habilidad social como el de Gambrill y otros (1975), el CSES, de Galassi y otros (1974) y el ASES (23) (24) (25). En base a ella se han construido otras versiones, como la RAS-M de Del Greco y otros (1981), para adolescentes, y la SRAS de McCormick (1985) para personas con bajo nivel lector (26) (27). Se ha informado de una buena fiabilidad test-retest (de 0.76 a 0.80 ), una buena consistencia interna (de 0.73 a 0.93 ) y validez concurrente (28) (29) (22) (30).

\section{c) Autoeficacia. Definición operacional e instrumento de medición.}

Para la medición cuantitativa de esta variable, se amplió a la Escala de Autoeficacia Generalizada, en su adaptación al español (31). El instrumento aporta rangos de calificación de los puntajes cuantitativos que se obtuvieron a través de la sumatoria del puntaje de cada ítem de la prueba. Según la validación de esta prueba en población chilena, la media del puntaje obtenido por los sujetos es 34,18 , siendo la desviación estándar de 4,84, por lo que los rangos, en este caso, se establecen en un margen bien acotado de puntuaciones (32).

La confiabilidad por consistencia interna de este instrumento, se ha obtenido en muestras de varios países, obteniendo coeficientes de alfa de Cronbach que van desde 0.76 a 0.90 . En cuanto a la validez, en estudios correlacionales se han encontrado relaciones positivas con emociones favorables, optimismo disposicional, y satisfacción con el trabajo (33). Los autores antes mencionados han obtenido un alfa de Cronbach de 0.78. Sin embargo, en su adaptación al español específicamente, muestra una consistencia de 0,87 (32).

\section{d) Rendimiento académico. Definición conceptual y operacional.}

Dada la complejidad del concepto, tomamos la definición de Pizarro (15) quien define al rendimiento académico como: "una medida de las capacidades respondientes o indicativas que manifiesta, en forma estimativa, lo que una persona ha aprendido como consecuencia de un proceso de instrucción o formación”. 
Esta es una variable cuantitativa que se basó en el promedio de calificaciones que presentaron los alumnos de la muestra, al cierre del año académico 2010. Así, se obtiene un valor numérico de 1 a 7; clasificando a partir del dígito 4, en orden numérico ascendente, dentro del rango de aprobación.

\section{RESULTADOS}

Para el análisis de los datos se usó el software SPSS en su versión 17.0, que permitió no sólo analizar los datos que se le proveyeron en función de los estadígrafos más comunes, sino además, construir gráficos y tablas que ayudaron a la comprensión y representación de los resultados obtenidos.

\section{Resultados que permiten describir a la muestra}

Se pudo describir que el grupo evaluado tiene una media de edad de 20 años, con una edad máxima de 27 y una mínima de 18 años; y que el 90,8 \% de la muestra se encuentra entre los 18 y 21 años de edad.

Respecto al sexo, encontramos que de los encuestados, el 83,7\% corresponde a mujeres, lo que es bastante esperable de acuerdo a la carrera que cursan.

De acuerdo a instrumento utilizado, el $62,8 \%$ de los sujetos tuvo puntuaciones correspondientes a un nivel alto de autoestima, el 32,6\% en un rango medio, y el 4,7\% en rango bajo.

En el caso de la asertividad, el 46,5\% de los estudiantes puntuó en rango alto, el 27,9\% de ellos en rango medio, y el $25,6 \%$ en rango bajo.

Acerca de la autoeficacia, la muestra se caracterizó por no tener sujetos en el rango sobre lo normal, evidenciándose un $72,1 \%$ de la muestra en los parámetros de la normalidad y un $27,9 \%$ de los sujetos en rango bajo lo normal.

Para un mejor análisis de los datos referentes al rendimiento académico, esta variable se transformó en una de tipo ordinal, definiendo tres categorías, de acuerdo al promedio de calificaciones obtenido por cada individuo. Para ello se siguió el criterio de fraccionar el rango total de valores obtenidos en esta muestra, en 3 intervalos iguales, que se definieron de la siguiente manera:

Rango $=($ Nota máx. - Nota Min $) / 3=(5,9-4,6) / 3=0,43 \sim 0,4$

De esta forma, los rangos definidos para la variable fueron:

$$
\begin{array}{ll}
\text { 1. } & 4,6 \leq x<5,0 \\
\text { 2. } & 5,0 \leq X<5,4 \\
\text { 3. } & 5,4 \leq X \leq 5,9
\end{array}
$$

De acuerdo a lo anterior, los resultados revelan que la minoría de la muestra $(18,6 \%)$ se encuentra en el rango 1 (de 4,6 a 4,9); que el 55,8\% de los estudiantes tiene puntuaciones entre 5,0 y 5,3; y que el $25,6 \%$ de los sujetos que conformaron la muestra tuvo calificaciones entre 5,4 y 5,9 . 


\section{Resultados que permiten inferir características de la muestra}

Para establecer la intensidad de la relación entre las variables, se usó el coeficiente de correlación de Spearman ( $\rho$ ), como es recomendado para variables de tipo ordinal. (19). Para la obtención de los resultados se selecciona las variables que presentan un valor $\mathrm{p} \leq$ 0.05 de significación; o un valor cercano a 1 en el coeficiente de Spearman, ya que las mediciones de este índice fluctúan de $+1 \mathrm{a}-1$, pasando por el cero, siendo este último valor un indicador de que no existe correlación entre las variables estudiadas, mientras que los dos primeros revelan la correlación máxima. Para el análisis de la significación entre variables, se utiliza la prueba de Wilcoxon como alternativa a la prueba $\mathrm{t}$ de Student, considerando el tamaño de la muestra y el tipo de variable (ordinal). Esta prueba permite comparar las medianas de dos muestras relacionadas y comprobar si existen diferencias entre ellas. Este estadígrafo es más sensible que el análisis de las correlaciones.

Seguidamente, la tabla I muestra los resultados de la aplicación de la prueba de Wilcoxon.

\begin{tabular}{|c|c|c|c|c|}
\hline \multicolumn{5}{|c|}{ Wilcoxon Signed Ranks Test } \\
\hline & & $\mathrm{N}$ & Mean Rank & Sum of Ranks \\
\hline \multirow[t]{4}{*}{ As ertividad - Autoestima } & Negative Ranks & $16^{a}$ & 9,63 & 154,00 \\
\hline & Positive Ranks & $2^{b}$ & 8,50 & 17,00 \\
\hline & Ties & $25^{c}$ & & \\
\hline & Total & 43 & & \\
\hline \multirow[t]{4}{*}{ As ertividad - Autoeficacia } & Negative Ranks & $5^{d}$ & 16,00 & 80,00 \\
\hline & Positive Ranks & $26^{\mathrm{e}}$ & 16,00 & 416,00 \\
\hline & Ties & $12^{f}$ & & \\
\hline & Total & 43 & & \\
\hline \multirow[t]{4}{*}{ Promedios - Autoestima } & Negative Ranks & $23^{9}$ & 14,93 & 343,50 \\
\hline & Positive Ranks & $5^{h}$ & 12,50 & 62,50 \\
\hline & Ties & $15^{i}$ & & \\
\hline & Total & 43 & & \\
\hline \multirow[t]{4}{*}{ Promedios - Autoeficacia } & Negative Ranks & $6^{j}$ & 12,00 & 72,00 \\
\hline & Positive Ranks & $19^{k}$ & 13,32 & 253,00 \\
\hline & Ties & $18^{\prime}$ & & \\
\hline & Total & 43 & & \\
\hline \multirow[t]{4}{*}{ Promedios - As ertividad } & Negative Ranks & $17^{\mathrm{m}}$ & 15,06 & 256,00 \\
\hline & Positive Ranks & $12^{n}$ & 14,92 & 179,00 \\
\hline & Ties & $14^{\circ}$ & & \\
\hline & Total & 43 & & \\
\hline
\end{tabular}

Test Statistics ${ }^{c}$

\begin{tabular}{|l|r|r|r|r|r|}
\hline & $\begin{array}{c}\text { Asertividad - } \\
\text { Autoestima }\end{array}$ & $\begin{array}{c}\text { idad - } \\
\text { Autoefi }\end{array}$ & $\begin{array}{c}\text { Promedios - } \\
\text { Autoestima }\end{array}$ & $\begin{array}{c}\text { Promedios - } \\
\text { Autoeficacia }\end{array}$ & $\begin{array}{c}\text { Promedios - } \\
\text { Asertividad }\end{array}$ \\
\hline$Z$ & $-3,258^{\mathrm{a}}$ & $-3,772^{\mathrm{b}}$ & $-3,470^{\mathrm{a}}$ & $-2,694^{\mathrm{b}}$ &,$- 895^{\mathrm{a}}$ \\
Asymp. Sig. (2-tailed) &, 001 &, 000 &, 001 &, 007 &, 371 \\
\hline
\end{tabular}

a. Based on positive ranks.

b. Based on negative ranks .

c. Wilcoxon Signed Ranks Test 
La tabla II muestra los resultados obtenidos al correlacionar las variables en estudio, a través del coeficiente de Spearman.

\begin{tabular}{|c|c|c|c|c|c|c|}
\hline \multicolumn{7}{|c|}{ Correlations } \\
\hline & & & Autoestima & Asertividad & Autoeficacia & Promedios \\
\hline \multirow[t]{12}{*}{ Spearman's rho } & Autoestima & Correlation Coefficient & 1,000 & $614^{* *}$ &, $616^{\text {** }}$ & ,104 \\
\hline & & Sig. (2-tailed) & &, 000 &, 000 & ,509 \\
\hline & & $\mathrm{N}$ & 43 & 43 & 43 & 43 \\
\hline & Asertividad & Correlation Coefficient &, $614^{* *}$ & 1,000 &, $554^{* *}$ & ,089 \\
\hline & & Sig. (2-tailed) &, 000 & &, 000 &, 570 \\
\hline & & $\mathrm{N}$ & 43 & 43 & 43 & 43 \\
\hline & Autoeficacia & Correlation Coefficient &, $616^{* *}$ &, $554^{* *}$ & 1,000 & ,070 \\
\hline & & Sig. (2-tailed) &, 000 &, 000 & & ,656 \\
\hline & & $\mathrm{N}$ & 43 & 43 & 43 & 43 \\
\hline & Promedios & Correlation Coefficient & ,104 & ,089 & ,070 & 1,000 \\
\hline & & Sig. (2-tailed) &, 509 &, 570 & ,656 & \\
\hline & & $\mathrm{N}$ & 43 & 43 & 43 & 43 \\
\hline
\end{tabular}

${ }^{\star *}$. Correlation is significant at the 0.01 level (2-tailed).

\section{A. Relaciones entre variables personológicas}

Al analizar la relación entre la asertividad y la autoestima, y observar el valor del coeficiente de Spearman, vemos que corresponde a 0.614, con $\mathrm{p}<0.05$; por lo tanto, se concluyó que existe una relación significativa entre dichas variables. Este resultado fue confirmado por el obtenido en la prueba de Wilcoxon $\mathrm{p}<0.05)$.

En el análisis de la relación entre autoeficacia y asertividad, se puso de manifiesto que existe una relación significativa y directamente proporcional entre las variables mencionadas, pues el valor del coeficiente de Spearman es 0.554, con $\mathrm{p}<0.05$; además, $\mathrm{p}<0.05$ en la prueba de Wilcoxon.

\section{B. Relaciones de variables personológicas con rendimiento académico}

En el caso de la relación de la autoestima con el rendimiento académico, obtuvimos que si bien no se demuestra que existe una relación directamente proporcional entre la autoestima y el rendimiento académico (el valor del coeficiente de Spearman es 0.104, con $p>0.05)$, se revela una relación significativa entre ambas variables ( $p<0.05$ en la prueba de Wilcoxon). Dado lo anterior, se rechazó la hipótesis de investigación que hacía referencia a que a mayor autoestima, habría mayor rendimiento académico; sin embargo, el análisis revela una relación entre ambas variables, pero no podemos determinar de qué tipo es la misma.

Al respecto de la relación de la autoeficacia con el rendimiento académico, de acuerdo a los resultados obtenidos, no se demuestra que existe una relación directamente proporcional entre la autoeficacia y el rendimiento académico (el valor del coeficiente de Spearman es 0.070 , con $\mathrm{p}>0.05$ ), pero se revela una relación entre ambas variables ( $\mathrm{p}<0.05$ en la prueba de Wilcoxon); de esta forma, se rechazó la hipótesis de que a mayor autoeficacia, habría mayor rendimiento académico; pero el análisis revela una relación entre ambas variables, aunque no podemos determinar de qué tipo es la misma. 
Al relacionar la asertividad con el rendimiento académico, se evidenció que la relación entre la autoeficacia y el rendimiento académico no es significativa, ya que el valor de significación es superior a 0,05 al determinar el coeficiente de Spearman, y el valor de éste es de 0.089, demostrándose la ausencia de una relación entre ambas variables. Los resultados en la prueba de Wilcoxon confirmaron los anteriormente descritos.

\section{DISCUSIÓN}

En esta investigación se relacionan la autoeficacia y la autoestima con la asertividad, así como las tres anteriores con el rendimiento académico. Esto es debido, por una parte, a la importancia que las variables personológicas y las habilidades sociales poseen para el fututo laboral de los profesionales de la salud, tal como se documenta en varias investigaciones, (4) (34) (5) y por otra, a la inexacta capacidad predictiva del rendimiento académico con respecto al éxito profesional (34) (35).

Los niveles observados en la muestra para autoestima, asertividad y autoeficacia están por sobre lo normal en porcentajes mayores del $46 \%$ de la población; es decir que los conceptos sobre sí mismos y las habilidades comunicacionales de estos estudiantes destacan por sobre los límites de la normalidad, lo que prueba excelentes condiciones de las mismas en la actualidad contextual de este grupo.

Ahora bien, si se revisan los niveles de asertividad con respecto a autoestima y autoeficacia alcanzados por la población en estudio, aparece una relación significativa y a su vez directamente proporcional entre las tres variables; es decir, se pudo comprobar que a mayor asertividad, se logra un aumento en la autoestima y autoeficacia; y viceversa, verificando de esta manera las hipótesis $\mathrm{H}_{1}$ y $\mathrm{H}_{2}$. Esto revela la capacidad o habilidad de estos jóvenes, tal como se indica en el párrafo inicial, que de mantenerse a través del tiempo favorecerá la posibilidad de un desempeño ocupacional apreciable desde la perspectiva de las competencias comunicacionales, sociales y personológicas que su futuro rol profesional les exige.

En referencia a la relación entre el rendimiento académico de los estudiantes con el nivel descrito de autoestima, autoeficacia y asertividad, si bien no es posible validar las hipótesis $\mathrm{H}_{3}, \mathrm{H}_{4}$ y $\mathrm{H}_{5}$ se puede concluir que existe una vinculación significativa entre estas variables aunque no sea posible determinar, en este estudio en particular, el grado de tal relación.

Al respecto de la autoestima, la asertividad y la autoeficacia, se evidencia una correlación significativa y directamente proporcional. Este resultado es esperable desde el punto de vista teórico, considerando que la asertividad, por sus dimensiones sociales, puede ser un catalizador para el desarrollo del individuo, de forma que al tener adecuada interacción con quienes le rodean, la retroalimentación que recibe de su medio acerca de sí mismo es positiva, siendo una buena influencia sobre la construcción del autoconcepto, que involucra, entre otras dimensiones, la autoestima y la autoeficacia (6) (36). Asimismo, otros autores (11) comparten los resultados de su investigación, en la

que se evidencia que los estudiantes con calificaciones altas en autoestima, de igual forma las tienen en asertividad.

Al correlacionar las variables personológicas (autoestima y autoeficacia) y la asertividad con el rendimiento académico, se hallan varios estudios previos que muestran que 
existen relaciones significativas y directamente proporcionales (13) (10) (14). En el caso de esta investigación, se encuentran resultados interesantes: la autoestima y la autoeficacia no muestran una relación directamente proporcional con el rendimiento, pero las pruebas estadísticas aplicadas revelan que sí existe algún tipo de vinculación entre estas variables, no determinada en esta investigación por no ser el objetivo de la misma.

Lo anterior es coherente con los resultados que han encontrado otros investigadores, (7) quienes hallan que la autoestima y el rendimiento académico se relacionan significativamente, en su muestra. Otros investigadores (38) (39) obtuvieron resultados similares, y algunos de ellos (10) (11) describen que si un estudiante cree que no tiene capacidad suficiente para rendir bien en las evaluaciones a las que se somete, probablemente sus puntuaciones no sean altas.

Desde el punto de vista teórico, resulta lógico que la variable de autoeficacia correlacione de forma positiva con el rendimiento académico. Dado que justamente las personas con un alto grado de autoeficacia se plantean metas más elevadas y son más perseverantes en su labor para conseguirlas, por lo que suelen lograr un mayor éxito que quienes tienen un grado de autoeficacia bajo (12). En esta investigación el nivel de correlación no permite asegurar una relación directamente proporcional, pero sí muestra indicadores de que ambas variables están vinculadas en algún grado.

Caso distinto es el de la relación entre la asertividad y el rendimiento académico. En este punto, las correlaciones no son significativas en ninguna de las dos pruebas estadísticas usadas, por lo que no se evidencia relación entre estas variables, en la muestra estudiada.

Desde la perspectiva teórica, la influencia de esta habilidad comunicacional en los resultados académicos puede estar relacionada con la capacidad de los estudiantes para desempeñarse en situaciones de trabajo cognitivo que requieran interacción social. Por lo anterior, si las evaluaciones no consideran esta dimensión, pudiera verse influido el nivel de correlación entre la asertividad y el rendimiento académico.

De todos modos, aunque las investigaciones publicadas muestran heterogeneidad en cuanto a sus resultados, la mayoría de ellas registran una vinculación poco clara de las variables personológicas y comunicacionales con el rendimiento académico, y no logran aportar hallazgos al respecto de la existencia de relaciones significativas entre la asertividad y el rendimiento académico (10) (40) (14) (11). Esto es coincidente con lo encontrado en este estudio.

Concluyendo, si bien la relación entre la autoestima, la asertividad y la autoeficacia con el rendimiento académico no es directamente proporcional e hipótesis relacionadas con estas correlaciones son desechadas, hay indicadores de que existe una vinculación entre las variables autoestima y autoeficacia, por lo que si bien no se pueden confirmar las hipótesis, no queda claro que estas variables sean completamente independientes una de la otra.

Por otra parte, existen repercusiones al respecto de las asunciones que se pueden hacer sobre los procesos formativos y evaluativos, al interpretar los resultados de esta investigación. De acuerdo a lo que se ha definido al hablar de rendimiento académico, este consiste en una calificación numérica que debiera reflejar el conocimiento alcanzado, por un estudiante, como resultado de un proceso de enseñanza/aprendizaje. Dado lo anterior, se podría hipotetizar que estas habilidades no constituyen el foco de 
atención de los programas de formación; o que los procedimientos evaluativos no son representativos de los procesos de formación y que estos sí intencionan el desarrollo de estas variables.

Asimismo, si consideramos que el desarrollo de la personalidad es un predictor del éxito profesional (4) (34) y que la relación de las variables personológicas (autoestima, autoeficacia y asertividad), en este estudio, con el rendimiento, no lo es, se podría decir entonces que éste, no es un fiel predictor del éxito profesional, por lo que esta investigación aporta evidencia de la importancia y la necesidad de integrar ambas áreas de formación y estas aristas del desarrollo de los estudiantes, sobre todo considerando que estos profesionales en formación se desempeñarán en el área de la salud (34) (35).

Estos resultados pueden también interpretarse de forma muy positiva, en función de la calidad de la muestra, de acuerdo a los niveles de asertividad, autoeficacia y autoestima que mostraron, lo que, además, habla de las potencialidades de este grupo de estudiantes, para desempeñarse como profesionales de la salud. Pero sin ir en detrimento de esto, la evidencia obtenida es preocupante, en el sentido del cuestionamiento que genera acerca del necesario equilibrio en la formación de profesionales, entre el manejo conceptual y técnico, y el desarrollo de la personalidad, en función de la calidad de la educación.

En general, las metodologías que parecieran ser más efectivas en términos del desarrollo de las habilidades sociales, estarían relacionadas con la certeza en que el estudiante puede ser un agente activo en su desarrollo, tanto cognitivo como afectivo; por ejemplo, el aprendizaje basado en problemas (ABP) o el aprendizaje por proyectos (AP), son buenos exponentes de lo anterior, en tanto que, para cumplir con los objetivos evaluativos y formativos, los estudiantes tienen que conjugar sus habilidades cognitivas de búsqueda, selección, síntesis e integración de la información, con la capacidad de interactuar con otros, exponer y defender sus puntos de vista de forma asertiva, comunicarse adecuadamente, etc. Todo parece indicar que metodologías más academicistas, en las que el alumno aprende de forma individual y dirigida, apuntan de forma más escueta al logro del desarrollo integral de los estudiantes.

Es así que se propone integrar al currículum de formación profesional, un grupo de actividades, articuladas con el resto de los programas de formación, que permita el desarrollo de la personalidad de los estudiantes y así favorezca la sistematización de su formación integral; ya que se considera que en gran parte, el rechazo de las hipótesis relacionadas con rendimiento académico se explica considerando que en muchos casos, el desarrollo de las características personológicas y de comunicación social, se realizan sólo de acuerdo al interés y al sello propio de cada docente, transversalmente durante el desarrollo de las actividades curriculares; pero como no forman parte de los objetivos explícitos de cada programa, tampoco se reflejan en los procedimientos evaluativos.

Por último, con esta investigación se procura motivar futuras indagaciones de tipo explicativo, por un lado, que pueden establecer relaciones de causalidad entre algunas variables estudiadas, dado que ese no es el propósito de este estudio. Así como también, por otra parte investigaciones de tipo documental, cualitativas, para focalizar como objeto de estudio los programas y metodologías de enseñanza en relación al desarrollo de las competencias blandas en las carreras de salud.

Asimismo se considera relevante realizar la ampliación y profundización de este estudio, tanto para la carrera de Terapia Ocupacional, como para el resto de las carreras del área 
de la salud, dada la trascendencia que sus variables presentan, no sólo para el futuro desempeño laboral de dichos profesionales, sino además para su óptima salud psicofísica y sus consecuencias, y porque, por otra parte, posibilitaría la ampliación de la muestra.

Sin embargo, dentro de las recomendaciones que surgen producto de esta experiencia investigativa, no sólo podríamos quedarnos con la motivación para nuevas investigaciones, tal como acabamos de explicitar, sino que también se pueden inferir acciones directas tales como: potenciar el crecimiento de los estudiantes de esta muestra con un plan de seguimiento para el desarrollo de estas habilidades, ya evaluadas. Al igual que fomentar el incremento de competencias blandas como parte de la formación integral de los alumnos a través de un programa sistemático, que sea parte de la malla curricular para generar el desarrollo de las habilidades sociales necesarias para los perfiles de egreso de la carrera; así como también incluir estrategias e instrumentos de evaluación durante el proceso de las prácticas profesionales que incluyan las competencias blandas. Además de la creación de Talleres específicos de habilidades sociales en función de las necesidades y características del alumnado; bitácoras de desarrollo profesional, etc.; junto con fomentar el trabajo colaborativo para apuntar al incremento de habilidades sociales, con acciones de co-evaluación.

\section{REFERENCIAS BIBLIOGRÁFICAS}

(1) BERRÍOS, M.P., AUGUSTO, J.M. Y AGUILAR, M. Inteligencia emocional percibida y satisfacción laboral en contextos hospitalarios: Un estudio exploratorio con profesionales de enfermería. Index de Enfermería. 2006; 15:30-34.

(2) MARTÍNEZ, C. Consideraciones sobre inteligencia emocional. La Habana. Editorial Científico-Técnica, 2008.

(3) OPAZO, R. Biblioterapia para el desarrollo asertivo. Santiago de Chile: Instituto Chileno de Psicoterapia Integrativa, 2004

(4) ZEIDNER M., MATTHEWS G. Y ROBERTS R.D. Emotional intelligence in the workplace: A critical review. Applied Psychology: An International Review, 2004; 53: 371-399.

(5) GIL-MONTE, P.R. El síndrome de quemarse por el trabajo (Síndrome de Burnout) en profesionales de enfermería. Revista Eletrônica InterAção Psy, 2008; 1:19-33.

(6) ROMÁN, J. Estrés y Burnout en profesionales de la salud de los niveles primario y secundario de atención. Revista Cubana Salud Pública, 2003; 29:103-110.

(7) TSAOUSIS I. Y NIKOLAOU I. Exploring the relationship of emotional intelligence with physical and psychological health functioning. Stress and Health, 2005; 21:77-86.

(8) MILICIC, N. Creo en ti. La construcción de la autoestima en el contexto escolar. Segunda edición. Santiago de Chile: LOM Ediciones, 2001.

(9) BLANCO, A. Creencias de autoeficacia de estudiantes universitarios: un estudio empírico sobre la especificidad del constructo. Relieve, 2010; 16:1-28.

(10) EDEL, R. El rendimiento académico: concepto, investigación y desarrollo. Revista Electrónica Iberoamericana sobre Calidad, Eficacia y Cambio en Educación, 2003; $1: 1-15$.

(11) LEÓN, A., RODRÍGUEZ, C., FERREL, F. Y CEBALlOS, G. Asertividad y autoestima en estudiantes de primer semestre de la Facultad de Ciencias de la 
Salud de una universidad pública de la ciudad de Santa Marta (Colombia). Psicología desde el Caribe. Revista del programa de Psicología de Universidad del Norte, Colombia, 2009.

(12) FELDMAN, R. Psicología con aplicaciones en países de habla hispana. Sexta edición. México DF: Mac Graw Hill, 2006.

(13) CASO-NIEBLA, J. HERNÁNDEZ-GUZMÁN, L. Variables que inciden en el rendimiento académico de adolescentes mexicanos. Revista Latinoamericana de Psicología, 2007; 39:487-501.

(14) GARRIDO, E. DE LA C., ORTEGA, N., ESCOBAR, E. Y GARCÍA, R. Evaluación de la asertividad en estudiantes universitarios, con bajo rendimiento académico. Revista Científica Electrónica de Psicología ICSa-UAEH, 2010; 3:117.

(15) REYES, Y. Relación entre el rendimiento académico, la ansiedad ante los exámenes, los rasgos de personalidad, el autoconcepto y la asertividad, en estudiantes del primer año de Psicología de la UNMSM. Tesis para optar al título de Psicólogo. Universidad Nacional Mayor de San Marcos, Lima, Perú, 2003.

(16) HARGREAVES, A. Enseñar en la sociedad del conocimiento. Barcelona: Ediciones Octaedro, S.L., 2003.

(17) GUTIÉRREZ B., L (s.f.). Paradigmas Cuantitativo y Cualitativo en la Investigación Socioeducativa: Proyección y Reflexiones. Recuperado el 3 de marzo de 2011, de: http://biblioteca.idict.villaclara.cu/UserFiles/File/metodologia de investigacion/paradigmas cuantitativo y cualitativo.doc

(18) MENDOZA, R. Investigación cualitativa y cuantitativa. Diferencias y Limitaciones. Recuperado el 04 de marzo de 2011, de:

http://www.gycperu.com/descargas/005investigacion cuali cuanti diferencias y limitac.pdf

(19) HERNÁNDEZ S., R., FERNÁNDEZ C., C., Y BAPTISTA L., P. Metodología de la investigación ( $3^{\circ}$ ed.) México DF: Mac Graw Hill, 2003.

(20) BRINKMANN, H. Y GARCÉS, M. Adaptación para adultos del Inventario de Autoestima de Coopersmith. Ponencia VI Congreso de Nacional de Psicología, Santiago, Chile, 1999.

(21) LARA CANTÚ, M., VERDUZCO, A., ACEVEDO, M. Y CORTÉS, J. (1993). Validez y Confiabilidad del Inventario de Autoestima de Coopersmith para Adultos, en la población mexicana. Revista Latinoamericana de Psicología, 1993; 25:247-255.

(22) RATHUS, S.A. A 30-item schedule for assessing assertive behaviour. Behaviour Therapy; 1973; 4:398-406.

(23) GAMBRILL, E.D. Y RICHEY, C.A. An Assertion inventory for use in assessment and research. Behavior Therapy, 1975; 6:550-561.

(24) GALASSI, J. P., DELO, J. S., GALASSI, M. D. Y BASTIEN, S. The College Self-Expression Scale, A measure of assertiveness. Behavior Therapy, 1974; 5:165-171.

(25) GAY, M. L., HOLlANDSWORTH, J. G. Y GALASSI, J. P. An assertive inventory for adults. Journal of Counseling Psychology, 1975; 22:340-344. 
(26) DEL GRECO, L., BREITBACH, L. Y MCCARTHY, R. H. The Rathus Assertiveness Schedule Modified for early adolescents. Journal of Behavior Assessment, 1981; 3:321-328.

(27) MCCORMICK, I. A. A simple version of the Rathus assertiveness schedule. Behavioral Assessment, 1985; 7:95-99.

(28) BECK, J. G. Y HEIMBERG, R. G. (1983). Self report assessment of assertive behavior. A critical analysis. Behavior Modification, 1983; 7:451-487.

(29) ECHEBURÚA, E. Evaluación y tratamiento de la fobia social. Barcelona: Martínez Roca, 1995.

(30) PEARSON, J. C. A factor analytic study of the items in the Rathus Assertiveness Schedule and the Personal Report of Communication Apprehension. Psychological Reports, 1979; 45:491-497.

\section{AGRADECIMIENTOS}

A Claudia Muñoz, Directora de la Carrera Terapia Ocupacional de la Universidad San Sebastián, sede Santiago de Chile, por los espacios ofrecidos y su valiosísima colaboración.

A los alumnos que ingresaron en el año 2010 a la carrera Terapia Ocupacional de la Universidad San Sebastián, sede Santiago de Chile, por su colaboración con este estudio, su cercanía y su disposición. 